\title{
Coplanar Common Points in Non-centric Cameras
}

\author{
Wei Yang ${ }^{1}$, Yu Ji ${ }^{1}$, Jinwei $\mathrm{Ye}^{2}$, S. Susan Young ${ }^{2}$, and Jingyi $\mathrm{Yu}^{1}$ \\ 1 University of Delaware, Newark, DE 19716, USA \\ 2 US Army Research Laboratory, Adelphi, MD 20783, USA
}

\begin{abstract}
Discovering and extracting new image features pertaining to scene geometry is important to 3D reconstruction and scene understanding. Examples include the classical vanishing points observed in a centric camera and the recent coplanar common points (CCPs) in a crossed-slit camera [21]17]. A CCP is a point in the image plane corresponding to the intersection of the projections of all lines lying on a common 3D plane. In this paper, we address the problem of determining $\mathrm{CCP}$ existence in general non-centric cameras. We first conduct a ray-space analysis to show that finding the CCP of a $3 \mathrm{D}$ plane is equivalent to solving an array of ray constraint equations. We then derive the necessary and sufficient conditions for CCP to exist in an arbitrary non-centric camera such as non-centric catadioptric mirrors. Finally, we present robust algorithms for extracting the CCPs from a single image and validate our theories and algorithms through experiments.
\end{abstract}

\section{Introduction}

An important task in computer vision is to identify and then extract image features pertaining to scene geometry for reliable $3 \mathrm{D}$ reconstruction. A classical example is the vanishing point (VP) in centric (perspective) cameras: a VP in the image plane $\Pi$ corresponds to the intersection of a set of parallel lines in space. The VP and the direction of the lines form one-to-one correspondence and tremendous efforts [6:5|3|2] have been focused on scene reconstruction using the VP. Recent studies [20] have shown that VPs generally exist in non-centric cameras such as the crossed-slit [21]10], linear oblique [9], etc.

The VP, in essence, is the characteristics of line directions. In contrast, the recently proposed coplanar common point or CCP is the characteristics of positions: for a set of (oblique) lines lying on a 3D plane, will their images still intersect at a common pixel in the image plane? The answer is generally no for centric cameras as these lines will map to 2D lines with different directions and origins in the image plane, with a singular case when the plane also pass through the camera's Center-of-Projection (CoP). Surprisingly, CCP generally exists for some special non-centric cameras such as the crossed-slit (XSlit) camera [21]. Although 3D lines map to curves (e.g., hyperbolas) as shown in Fig. 1 these curves intersect at the CCP as far as they lie on the same plane. In fact, the $\mathrm{CCP}$ and the 3D plane forms one-to-one mapping, a highly useful property for $3 \mathrm{D}$ scene reconstruction and understanding.

In this paper, we address the problem of determining CCP existence in general noncentric cameras [13]. On the theory front, we conduct a comprehensive analysis in rayspace and show that finding the CCP of a 3D plane is equivalent to solving an array

D. Fleet et al. (Eds.): ECCV 2014, Part I, LNCS 8689, pp. 220-233, 2014.

(C) Springer International Publishing Switzerland 2014 

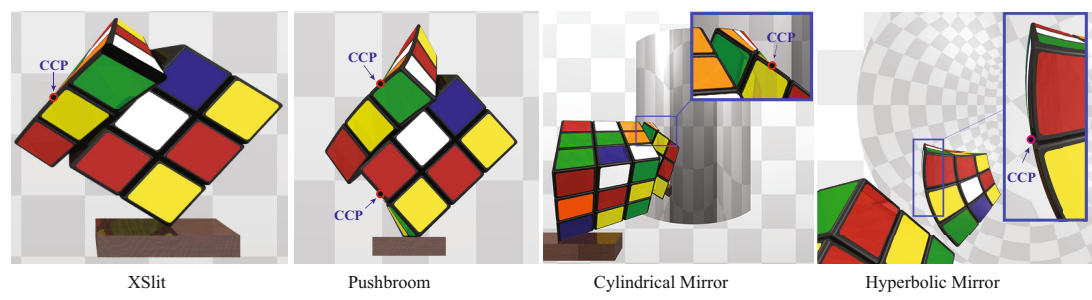

Fig. 1. CCPs in several non-centric cameras

of ray constraint equations. We then derive the necessary and sufficient conditions for $\mathrm{CCP}$ to exist in general non-centric cameras such as catadioptric mirrors. Our analysis further reveals the relationship between the CCP and the caustic (focal) surfaces of rays. Despite being largely theoretical, our framework finds its uses in 3D plane localization and reconstruction. Specifically, we develop robust algorithms for fitting curved images of 3D lines, locating the CCPs, and mapping them back to 3D planes. We validate our theory and algorithms on both synthetic data and real non-centric cameras, and we show that the CCP analysis provides useful insights on scene composition and configuration.

\section{Ray Space Analysis}

To characterize CCPs in an arbitrary non-centric camera, we conduct a ray space analysis. We describe a camera, centric or non-centric, as 2D manifold of rays, where each pixel on the sensor maps to a unique ray. Given a set of lines lying on a 3D plane $\Pi$, if their images in the camera pass through a CCP, then the CCP (which is a pixel) should map to a ray that lie on plane $\Pi$. We therefore can reformulate the problem of finding the $\mathrm{CCP}$ into finding the ray that satisfies a specific set of constraints.

Before proceeding, we explain our notations. To represent the ray space, we adopt the two-plane parametrization (2PP) model [7]. In 2PP, each ray is parameterized by its intersections with two parallel planes where $[s, t]$ is the intersection with the first plane $\Pi_{s t}$ and $[u, v]$ the second $\Pi_{u v}$. To simply our analysis, we choose the $z=0$ plane to be $\Pi_{u v}$, and $z=1$ plane to be $\Pi_{s t}$. Further, we use $\sigma=s-u$ and $\tau=t-v$ to parameterize the ray direction as $[\sigma, \tau, 1]$. All rays are parameterized as the 4-tuple $[u, v, \sigma, \tau]$.

Given a 3D plane with normal $\left[n_{x}, n_{y}, n_{z}\right]$ can be parameterized as $\Pi: n_{x} x+n_{y} y+$ $n_{z} z+d=0$, any ray $r[u, v, \sigma, \tau]$ lying on $\Pi$ should satisfy two constraints: 1$) r$ 's origin must lie on the plane and 2) $r$ 's direction is orthogonal to ח's normal. Therefore, we can derive the ray-on-plane constraints as:

$$
\left\{\begin{array}{l}
n_{x} u+n_{y} v+d=0 \\
n_{x} \sigma+n_{y} \tau+n_{z}=0
\end{array}\right.
$$

To find whether CCP exists in a general non-centric camera, we set out to combine the ray-on-plane constraints with the camera's ray constraints and determine if there exists a solution that satisfies all constraints. 


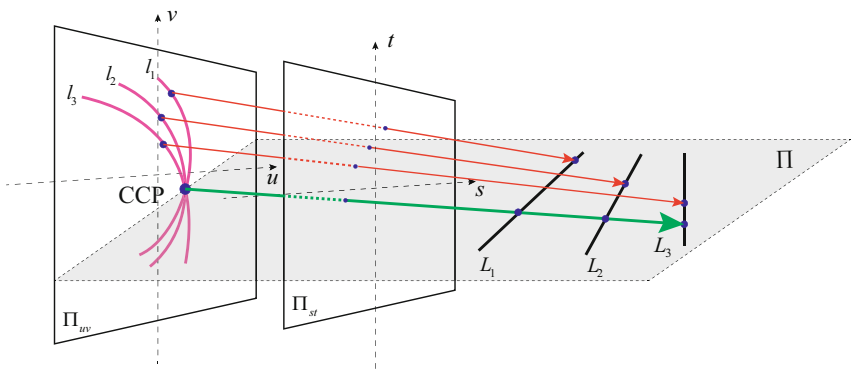

Fig. 2. CCP in $4 \mathrm{D}$ ray space. CCP is a pixel on the image plane corresponding to the intersection of the projections of all lines lying on a common 3D plane.

\subsection{CCPs in General Linear Cameras}

Yu and McMillan [19] introduced a class of primitive non-centric cameras called the general linear cameras or GLC. They correspond to 2D affine subspaces embedded in the 4D light field space [20] and they can be used to describe a broad range of commonly used non-centric cameras including pushbroom [4], XSlit, and linear oblique cameras.

A GLC is constructed by three generator rays $r_{1}, r_{2}, r_{3}$ so that all rays that collected by GLC are affine combinations of these three rays:

$$
G L C=\left\{r: r=\alpha r_{1}+\beta r_{2}+(1-\alpha-\beta) r_{3}, \forall \alpha, \beta\right\}
$$

where $\alpha, \beta$ are affine coefficients.

Without loss of generality, we can pick three special generator rays originating from $[1,0],[0,1]$ and $[0,0]$ on $\Pi_{u v}$ and rewrite the GLC equation as two linear constraints:

$$
\left\{\begin{array}{l}
\sigma=u \sigma_{1}+v \sigma_{2}+(1-u-v) \sigma_{3} \\
\tau=u \tau_{1}+v \tau_{2}+(1-u-v) \tau_{3}
\end{array}\right.
$$

where $\left[\sigma_{i}, \tau_{i}\right], i=1,2,3$ are the directions of the three ray generators.

Now that given a 3D plane $\Pi$, to determine if it has a CCP in the GLC, we can simply set out to find if there exists a ray that simultaneously satisfy the ray-on-plane constraints (Eqn. 1 and the GLC constraints (Eqn. 3). Notice that combining the two sets of equations result in a $4 \times 4$ linear system in $[u, v, \sigma, \tau]$ :

$$
\left[\begin{array}{cccc}
-n_{x} & -n_{y} & 0 & 0 \\
0 & 0 & -n_{x}-n_{y} \\
\sigma_{3}-\sigma_{1} & \sigma_{3}-\sigma_{2} & 1 & 0 \\
\tau_{3}-\tau_{1} & \tau_{3}-\tau_{2} & 0 & 1
\end{array}\right]\left[\begin{array}{c}
u \\
v \\
\sigma \\
\tau
\end{array}\right]=\left[\begin{array}{c}
d \\
n_{z} \\
\sigma_{3} \\
\tau_{3}
\end{array}\right]
$$

Whether the linear system has a solution depends on the determinant $J$ :

$$
J=n_{x}^{2}\left(\sigma_{2}-\sigma_{3}\right)+n_{x} n_{y}\left(\tau_{2}-\tau_{3}-\sigma_{1}+\sigma_{3}\right)-n_{y}^{2}\left(\tau_{1}-\tau_{3}\right)
$$


By solving the linear system (Eqn. 4), the CCP coordinate on the image plane can be computed as:

$$
\left\{\begin{array}{l}
u=\frac{1}{J}\left[n_{y}\left(n_{x} \sigma_{3}+n_{y} \tau_{3}+n_{z}\right)-d \cdot m_{2}\right] \\
v=\frac{1}{J}\left[d \cdot m_{1}-n_{x}\left(n_{x} \sigma_{3}+n_{y} \tau_{3}+n_{z}\right)\right]
\end{array}\right.
$$

Where $m_{1}=n_{x}\left(\sigma_{1}-\sigma_{3}\right)+n_{y}\left(\tau_{1}-\tau_{3}\right), m_{2}=n_{x}\left(\sigma_{2}-\sigma_{3}\right)+n_{y}\left(\tau_{2}-\tau_{3}\right)$. Now that let us look at specific types of GLCs. To simplify our analysis, we assume that we translate the $u v$ plane so that the third generator ray passes both the origins of the $s t$ and $u v$ plane, i.e., $r_{3}=[0,0,0,0]$.

Pinhole: Assume the camera's CoP is at $[0,0, f]$, by using the similitude relationship, we have $\sigma_{2}=\sigma_{3}=0, \tau_{1}=\tau_{3}=0, \sigma_{1}-\sigma_{3}=\tau_{2}-\tau_{3}=-1 / f$. Therefore, we have $J=0$ for any plane $\Pi$. Hence, CCPs do not exist in a pinhole camera.

Pushbroom: A pushbroom camera collects rays that pass through a common slit and parallel to a plane. We assume that the slit is parallel to $\Pi_{u v}$ at distance $Z$. It is easy to see that we have $\sigma_{2}=\sigma_{3}=0, \tau_{1}=\tau_{3}=0, \sigma_{1}-\sigma_{3}=0, \tau_{2}-\tau_{3}=-1 / Z$. Therefore, $J \neq 0$ for a general 3D plane and CCPs exist in a pushbroom camera.

XSlit: A XSlit camera collects rays that pass through two slits. We assume the two slits are orthogonal and both parallel to $\Pi_{u v}$. The horizontal and vertical slits are at depth $Z_{1}$ and $Z_{2}$ respectively. We have $\sigma_{2}=\sigma_{3}=0, \tau_{1}=\tau_{3}=0, \sigma_{1}-\sigma_{3}=-1 / Z_{2}$, $\tau_{1}-\tau_{3}=-1 / Z_{1}$. Therefore, $J \neq 0$ for a general 3D plane and CCPs exist in XSlit cameras.

Pencil and Bilinear: If we assume that the slit is parallel to $\Pi_{u v}$ at depth $Z$, we have $\sigma_{2}=1 / Z, \sigma_{3}=0, \tau_{1}=\tau_{3}=0, \sigma_{1}-\sigma_{3}=-1 / Z, \tau_{2}-\tau_{3}=-1 / Z$. Therefore, $J \neq 0$ for a general 3D plane and therefore CCPs exist. A similar conclusion holds for the bilinear (linear oblique) [9] cameras. The complete results are showed in Table. 1 .

Table 1. CCP existence in popular GLCs

\begin{tabular}{cccccc}
\hline \multicolumn{4}{c}{ Pinhole XSlit Pushbroom Pencil Bilinear } \\
\hline $\begin{array}{c}\text { CCP } \\
\text { Existence }\end{array}$ & $\times$ & $\sqrt{ }$ & $\sqrt{ }$ & $\sqrt{ }$ & $\sqrt{ }$ \\
\hline
\end{tabular}

Our analysis reveals that the pinhole camera is one of the very few singular cases where a 3D general does not exhibit a CCP. Notice that many of the GLC models have real world implementations. For example, pushbroom and XSlit and pencil images can be captured by a rolling shutter camera [8] or synthesized by stitching specific rows or columns from a row of pinhole images, as shown in Section 4.2. Therefore, CCPs can be potentially benefit scene reconstruction using these cameras.

\subsection{Concentric Mosaics}

There are other widely used non-centric cameras, for example, concentric mosaics [16 12] or circular XSlit [22]. These camera models are generally synthesized from 


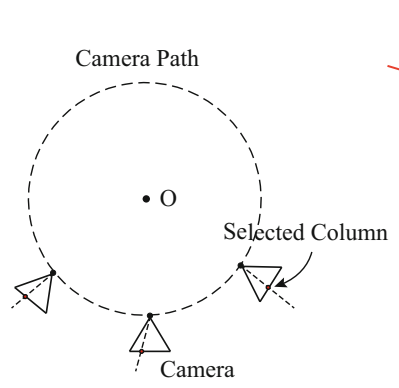

(a)

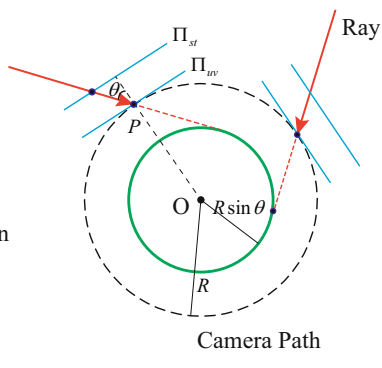

(b)

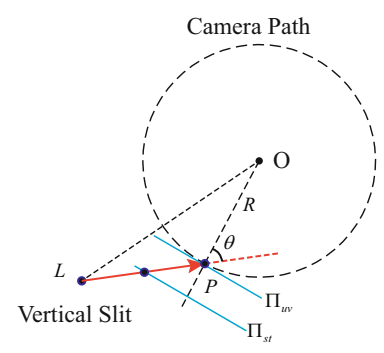

(c)

Fig. 3. (a) Concentric mosaics are synthesized from a sequence of images captured by a perspective camera moving along a circular path. (b) We define 2PP tangent to the camera path and rotating with the camera. (c) Circular XSlit panorama.

rotational panoramas. To acquire a concentric mosaics, it is common practice to rotate a camera off-axis on a circle. For each camera position, a column is sampled according to its angle from the optical axis. Then all selected columns are stitched together to form a panorama, as showed in Fig. 3] a).

To investigate the existence of CCP, we set the origin of the coordinate system as the rotation axis. Assume the $x y$ plane is the the camera path plane and we can also adopt 2PP model for ray parametrization. However instead of using two fixed planes, we use two parallel planes that rotate along with the camera. We set $\Pi_{u v}$ tangent to the camera path at $\mathrm{P}$ and vertical to the $x y$ plane. $\Pi_{s t}$ is parallel to $\Pi_{u v}$ with distance 1 . At each position, the column with angle $\theta$ is sampled. The set of all collected ray intersect $\Pi_{s t}$ at $[x+(x-y \tan \theta) / R, y+(y+x \tan \theta) / R]$. This allows us to map rays collected by the camera as a $2 \mathrm{D}$ manifold defined by $x, y$ and $\theta$ :

$$
[u, v, \sigma, \tau]=[x, y,(x-y \tan \theta) / R,(y+x \tan \theta) / R]
$$

Notice the $z$ value of the intersection on $\Pi_{s t}$ is not necessarily 1 as in the conventional 2PP case. Assume $z=\lambda$, we can rewrite the ray-on-plane constraints as:

$$
\left\{\begin{array}{l}
n_{x} x+n_{y} y+d=0 \\
\sin \theta(\sin \theta x+\cos \theta y) \cdot n_{x}+\sin \theta(\sin \theta y-\cos \theta x) \cdot n_{y}+\lambda n_{z}+d=0
\end{array}\right.
$$

Notice though that the solution to Eqn.7 is actually the intersection of $\Pi$ and a cylinder centered at origin and with radius $R \sin \theta$. Therefore Eqn.7 essentially indicates that the plane should have intersections both with the camera path circle and the cylinder, as showed in Fig. 3 b). This analysis is consistent with the geometric interpretation: the cylinder is the inner viewing surface and all rays the collected by the camera should be tangent to the cylinder. This type of concentric mosaics resembles the pushbroom camera where the central columns are stitched together.

A different way to construct concentric mosaic is proposed in [22], analogous to stitching an XSlit panorama from a translational array of images. Using the same acquisition setup, we select, at each camera position, the column with the ray that passes a 
predefined vertical slit, as showed in Fig 3 (c). The result is a circular XSlit model with one vertical slit and one circular slit where the circular slit is the trajectory of the camera. In this set up, $\theta$ is a nonlinear function of $x$ and $y$. The intersections of the rays collected by $\mathrm{P}$ and $\Pi_{s t}$ is along PL. Hence $\sigma=\widetilde{\lambda}\left(s_{x}-x\right), \tau=\widetilde{\lambda}\left(s_{y}-y\right), s_{x}, s_{y}$ are $x$ and $y$ coordinates of the vertical slit. Eqn.7 nnow becomes: $n_{x} \widetilde{\lambda}\left(s_{x}-x\right)+n_{y} \widetilde{\lambda}\left(s_{y}-y\right)+\lambda n_{z}=$ 0 . Since $\lambda, \widetilde{\lambda}$ are both scalers, we can eliminate $\widetilde{\lambda}$ as:

$$
\left\{\begin{array}{l}
n_{x} x+n_{y} y+d=0 \\
n_{z} s_{x}+n_{z} s_{y}+\lambda n_{z}+d=0
\end{array}\right.
$$

This indicates that for a plane $\Pi$ to have a CCP, it should intersect with both the vertical slit and the camera path.

\section{CCPs in Catadioptric Mirrors}

A commonly used class of real non-centric cameras are catadioptric mirrors [1]15] in which a regular pinhole camera is positioned in front of a curved mirror for acquiring images with much wider field-of-view (FoV).

\subsection{Ray Space vs. Caustics}

Recall that our goal is to determine if we can find an incident ray collected by the camera that lies on $\Pi$. Notice that each point $P(x, y, z)$ on the mirror surface corresponds to a reflection ray. Hence we can also potentially map the CCP problem into the ray space similar to the GLC and concentric mosaic case: assume the mirror surface is in form $z(x, y)$, the incident ray $\mathbf{v}_{i}=\left[i^{x}, i^{y}, 1\right]$ can be computed as:

$$
\mathbf{v}_{i}=\mathbf{v}_{r}-2 \frac{\mathbf{n}_{s} \cdot \mathbf{v}_{r}}{\left\|\mathbf{n}_{s}\right\|^{2}} \mathbf{n}_{s}
$$

$\mathbf{n}_{s}$ is the surface normal and $\mathbf{v}_{r}$ is the reflection ray. Intersect this ray with $\Pi_{u v}$ and $\Pi_{s t}$, we can get the $4 \mathrm{D}$ representation:

$$
[u, v, \sigma, \tau]=\left[x-z \cdot i^{x}, y-z \cdot i^{y}, i^{x}, i^{y}\right]
$$

$u, v, \sigma, \tau$ are functions in $x$ and $y$. The set of rays collected by the mirror surface form a ray-space parametric manifold in $x$ and $y$.

$$
\Sigma(x, y)=[u(x, y), v(x, y), \sigma(x, y), \tau(x, y)]
$$

We have the ray-on-plane constraints:

$$
\left\{\begin{array}{l}
u(x, y) n_{x}+v(x, y) n_{y}+d=0 \\
\sigma(x, y) n_{x}+\tau(x, y) n_{y}+n_{z}=0
\end{array}\right.
$$

For a given plane $\Pi$, we have two equations, two unknowns. In theory, we can determine if $\Pi$ has a CCP by testing if Eqn. 12 has a solution. In reality, $\Sigma(x, y)$ can become highly complex and searching for the solution is a challenging algebraic problem. 
A different and more intuitive solution is to view the problem from the caustic perspective. Caustic is a curve or surface where the light rays concentrate[14]. The caustic surface can be computed using Jacobian method which results in a quadratic equation. A quadratic equation generally produces two solutions, hence the caustic surfaces always appear in pairs and locally they can be interpreted as XSlit[18]. If a plane has CCP, then the plane has to intersect with the two caustic surfaces, a necessary condition for $\mathrm{CCP}$ to exist. However, the condition is insufficient: the resulting intersections are two curves on the caustic surfaces. Since the caustic surfaces need to appear in pairs, the two curves do not necessarily form valid correspondences. Therefore, we would need to check for, every ray originating from the first curve, whether it will pass through the second curve. If there exists such a ray, then CCP exists. Otherwise, it does not. The procedure above provides a simple but effective recipe for determining CCP existence. In the following sections, we analyze several commonly used catadioptric mirrors.

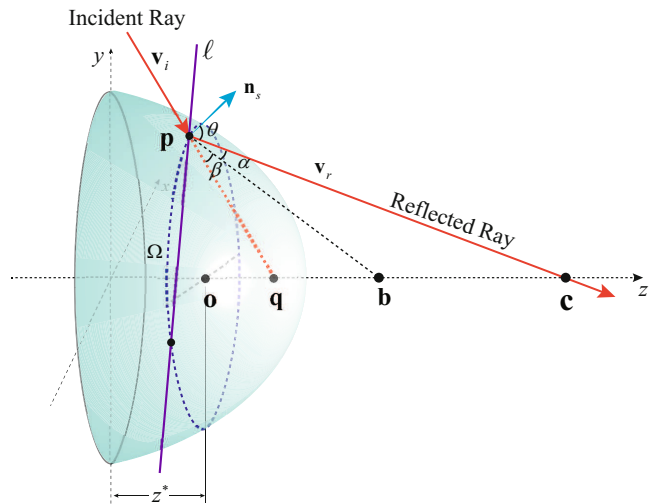

(a)

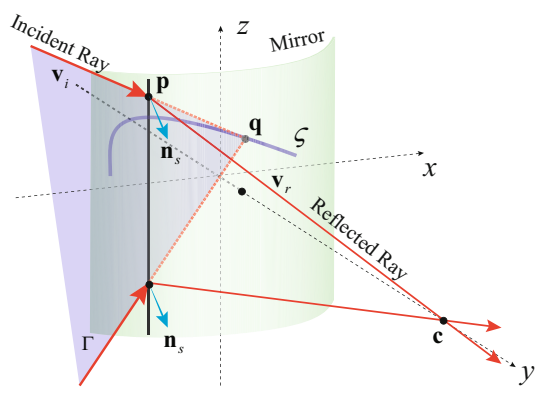

(b)

Fig. 4. (a) CCP condition in a rotationally symmetric mirror: the plane has to intersect with $z$ axis at a point $\mathbf{q}$ and have intersections with the $\Omega$ that determined by $\mathbf{q}$. (b) CCP condition in a cylinder mirror: the plane has to intersect with the caustic $\zeta$ and not perpendicular to the $x y$ plane.

\subsection{Rotationally Symmetric Mirrors}

A rotationally symmetric mirror is formed by rotating a quadric curve about its symmetric axis. Assume the symmetric axis is aligned with the $\mathrm{z}$-axis, the mirror surface can be parameterized as

$$
r^{2}+A z^{2}+2 B z-C=0, x^{2}+y^{2}=r^{2}
$$

$A, B$ and $C$ are the curve parameters that determine the mirror shape. In particular, $A=1, B=0, C>0$, the mirror is a sphere; $A>0, C>0$, elliptical mirror; and $A<0, C>0$, hyperbolic mirror. We assume that the pinhole viewing camera is on the symmetric axis of the mirror. The only singular case is when the pinhole coincides with 
curve's foci that emulates a virtual pinhole, in which the CCP does not exist. Therefore, in order to observe the CCP, we need to place the viewing camera off the foci.

By the symmetry, all reflection rays that collected by the view camera should intersect the symmetric axis. Hence the the symmetric axis is one of the two caustic surfaces.

CCP Condition 1: The 3D plane must have an intersection with the symmetric axis.

The projection under a rotationally symmetric mirror is shown in Fig. 4(a). Given an incident ray $\mathbf{v}_{i}$ from the scene towards the mirror surface, to produce image in the viewing camera, its reflection ray $\mathbf{v}_{r}$ must pass through the $\operatorname{CoP} \mathbf{c}=[0,0, c]$, lying on the $\mathbf{z}$-axis. Since $\mathbf{v}_{i}$ and $\mathbf{v}_{r}$ are coplanar, $\mathbf{v}_{i}$ also have an intersection with the $\mathbf{z}$-axis. Therefore, a valid CCP projection must intersects with the $\mathrm{z}$-axis, and hence the plane.

As mentioned above, the plane that intersects with the symmetric axis does not necessarily have a CCP. Assume $\mathbf{q}=\left[0,0, d^{\prime}\right], d^{\prime}=-d / n_{z}$ is the intersection point between the common plane and symmetric axis. We now only need to check the set of rays determined by $\mathbf{q}$. Assume the incident ray is reflected at $\mathbf{p}$ on the mirror surface and we have $\mathbf{v}_{i}=\mathbf{p}-\mathbf{q}$. It is important to note that ruled by the law of reflection, there only exists one circle of $\mathbf{p}$ on the mirror whose resulting reflection ray can enter c. Assume the circle is $\Omega: x^{2}+y^{2}=r^{* 2}$ for $\mathbf{p}$, we can obtain the set of rays as a cone that connects $\mathbf{q}$ and $\Omega$.

To compute $\Omega$, we orthogonally project $\mathbf{p}$ onto the $\mathrm{z}$-axis and obtain $\mathbf{o}=\left[0,0, z^{*}\right]$. By Eqn. 13, the surface normal at $\mathbf{p}$ can be computed as $\left[x^{*}, y^{*}, A z^{*}+B\right]$. Thus the tangent plane at $\mathbf{p}$ is $x^{*} x+y^{*} y+\left(A z^{*}+B\right) z+\left(B z^{*}-C\right)=0$. Hence we can compute the intersection point $\mathbf{b}$ of the tangent plane and z-axis. Since $\forall p \in \Omega$, the corresponding reflection rays pass through $\mathbf{c}$, the tangent plane bisect the angle formed by $\mathbf{v}_{i}$ and $\mathbf{v}_{r}$, i.e, $\alpha=\beta=90^{\circ}-\theta$, by the law of reflection, as shown in Fig. 4 . Consider the triangle formed by $\mathbf{q}, \mathbf{p}$ and $\mathbf{c}$, we can formulate the following equation by applying the angle bisector theorem to solve for $z^{*}$

$$
\frac{\sqrt{r^{* 2}+\left(d^{\prime}-z^{*}\right)^{2}}}{\sqrt{r^{* 2}+\left(c-z^{*}\right)^{2}}}=\frac{C-B d^{\prime}-\left(B+d^{\prime} A\right) z^{*}}{(c A+B) z^{*}+c B-C}
$$

The solution to Eqn. 14 corresponds to valid reflection points on the mirror surface.

CCP Condition 2: The 3D plane must have intersection with $\Omega$.

Recall that not all the planes contain $\mathbf{q}$ will intersect with the circle $\Omega$. To test the plane-circle intersection, we compute the distance from the plane to the $\mathrm{z}$-axis at $z=z^{*}$ as:

$$
D=\frac{\left|n_{z}\left(z^{*}-d^{\prime}\right)\right|}{\sqrt{n_{x}^{2}+n_{y}^{2}}}
$$

If $D>r^{*}$, the plane will have no intersection with the circle and thus has no CCP. If $D=r^{*}$, the plane has one intersection with the circle and thus has a single CCP; When $D<r^{*}$, the plane has two intersections with the circle and will have two CCPs.

Plane Reconstruction from CCPs. In rotationally symmetric mirrors, a plane can be directly located from its CCPs. Recall that a plane may have one or two CCPs. We consider the two cases separately. 
Case I: One CCP. If there is only one CCP, the plane must be the tangent plane of the circle $\Omega$. Therefore, we can first find the tangent line $l$ at $z=z^{*}$ of $\Omega$. The plane can be reconstructed through the $l$ and the CCP's corresponding q.

Case II: Two CCPs. If two CCPs exist, we can instantly recover the plane by three points on it, i.e, the two CCPs and their corresponding $\mathbf{q}$.

\subsection{Cylinder Mirrors}

Another commonly used class of catadioptric mirrors is cylinder mirrors. Given a quadratic curve on the xy-plane, instead of rotating it about its symmetric axis, we extrude the curve along the z-axis to form a cylinder. By aligning the y-axis with symmetric axis, a cylinder mirror can be parameterized as

$$
x^{2}+A y^{2}+2 B y-C=0, z=t
$$

Same as the rotational symmetric mirror, $A, B$ and $C$ are the quadratic curve parameters. When $A=1, B=0, C>0$, we have a cylindrical mirror; $A>0, C>0$, elliptical cylinder mirror; and $A<0, C>0$, hyperbolic cylinder mirror. We place the camera on the $y$-axis such that the CoP can be written as $\mathbf{c}=[0, c, 0]$.

Whether the cylinder mirrors have one dimensional caustic is not as clear as the rotationally symmetric mirrors. We start with considering the forward projection problem, i.e, finding the incident ray constraints whose corresponding reflection ray will pass through the CoP, as shown in Fig. 4 b). Assume $\mathbf{p}=[x, y, z]$ is a point on the mirror surface where reflection occurs. First, we can determine the direction of the reflection ray $\mathbf{v}_{r}$ by connecting $\mathbf{p}$ and $\mathbf{c}$. Thus we have $\mathbf{v}_{r}=[-x,-y, c-t]$. The surface normal at $\mathbf{p}$ can be computed as $\mathbf{n}_{s}=[x, A y+B, 0]$. By specular reflection, we can compute the incident ray from Eqn. 9 as:

$$
\mathbf{v}_{i}=\left[\frac{2 g-f}{f} x, \frac{2 A g-f}{f} y+\left(\frac{2 g}{f} B+c\right),-t\right]
$$

Where $f(x)=(1-A) x^{2}+\left(B^{2}-A C\right), g(y)=-(B+A c) y+(B c-C)$. The incident ray can be parameterized in the point-direction form as $\mathbf{p}+\lambda \mathbf{v}_{i}$. Let $\lambda=1$, we have the intersection point of the incident ray and the xy-plane $(z=0)$ as $\mathbf{q}=$ $[2 g x / f, 2 g(A y+B) / f+c, 0]$. Notice that $\mathbf{q}$ is independent of the $z$ component of p. This indicates that for all $\mathbf{p}$ on a vertical line (parallel to $z$-axis) on the mirrors surface, the corresponding effective incident ray will pass through the same point $\mathbf{q}$ on $\mathrm{xy}$-plane. From the geometric perspective, $\mathbf{q}$ is actually the reflection point of the CoP w.r.tthe tangent plane of the mirror surface at $\mathbf{p}$ and hence it is equivalent to a virtual CoP. Since all points on a vertical line share the same tangent plane, the CoP reflection $q$ remains the same. Further, by sliding the vertical line on the mirror surface, we obtain a set of $\mathbf{q}$ that form a curve $\zeta . \zeta$ is the one dimensional caustic of cylinder mirrors and can be derived in $\mathbf{q}$ as:

$$
\zeta(x, y)=\Sigma\left\{\left[\frac{2 g x}{f}, 2 \frac{g}{f}(A y+B)+c, 0\right]\right\}
$$

For all $x, y$ on the cylinder mirror surface. 
CCP Condition: the 3D plane must intersect with $\zeta$.

As mentioned before, each point on $\zeta$ determines a set of rays on a vertical plane $\Gamma$, as show in Fig. 4 (b). If only the common plane does not perpendicular to the $\mathrm{x}-\mathrm{y}$ plane, i.e $n_{z} \neq 0$, there will be one valid CCP projection ray which is the intersection line of the common plane and $\Gamma$. Similar to the rotationally symmetric mirror example, the number of intersections between the common plane and $\zeta$ determines the number of CCPs.

\section{Experiments}

To validate our theory and analysis, we experiment on both synthetic and real data.

\subsection{Synthetic Experiment}

We render catadioptric mirror images using the POV-Ray ray tracer (www.povray.org). Our first experiment is performed on a cylindrical mirror $x^{2}+(y-10)^{2}=16, z=t$ where the viewing camera is place at the origin $(0,0,0)$ facing towards the cylindrical mirror. Our scene consists with two planes: $\Pi_{1}: 2.7475 y+z-12=0$ and $\Pi_{2}$ : $3.9153 y+z-8.9378=0$. Each plane consists of five lines, among which three are parallel. The camera and scene setups are shown in Fig.5(a). As shown in Fig. 5(b), $\Pi_{1}$ has one intersection with $\zeta$ while Plane $\Pi_{2}$ has two. Fig. 5 c) shows the captured catadioptric images of $\Pi_{1}$ and $\Pi_{2}$ and we can observe that $\Pi_{1}$ has one $\mathrm{CCP}$ and $\Pi_{2}$ has two. Our results are hence consistent with the theoretical prediction.
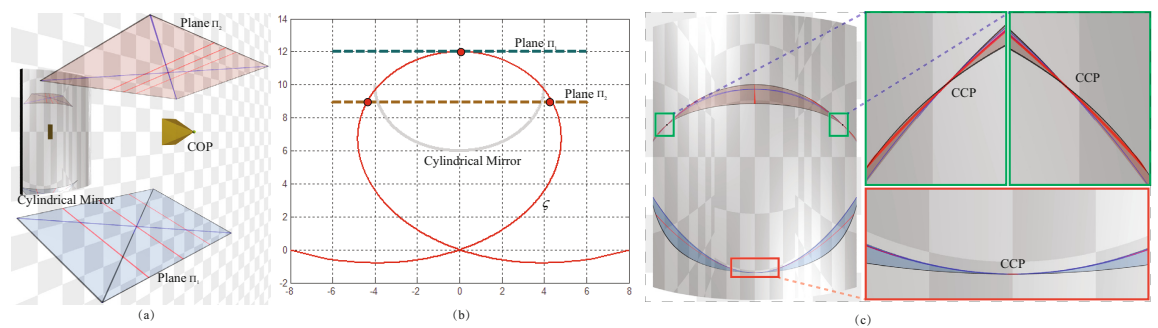

Fig. 5. Experiments on a cylindrical mirror. (a) Experimental setup; (b) Intersections between each plane and $\zeta$; (c) Rendered catadioptric image with close-up view at each CCP.

Next, we test on a hyperbolic mirror $z^{2} / 16-r^{2} / 9=1$ with the viewing camera the origin. The scene consists of two planes: $\Pi_{3}: 0.6608 y-z+5.1530=0$ and $\Pi_{4}: 0.7908 y+z-5.4184=0$. Same as our first experiment, we place five lines on each plane. The experimental setup is shown in Fig. 6a). Fig.6(b) shows the captured catadioptric image of the two planes and the numbers and position of CCP are consistent with the intersection points between the plane and $\Omega$ as predicted by our theory. We can further map the CCPs back to 3D space, showed in Fig. 6(c). The recovery of the 3D planes are highly accurate. 


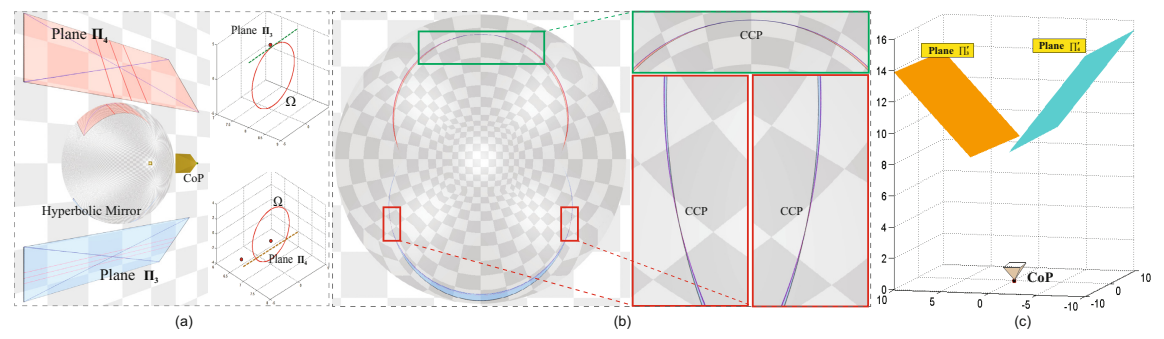

Fig. 6. Experiments on a hyperbolic rotationally symmetric mirror. (a) Experimental setup; (b) Rendered catadioptric image with close-up view at each CCP; (c) The recovered planes.

\subsection{Real Experiment}

Next, we conduct experiments on real non-centric cameras.

General Linear Cameras. We synthesize several popular GLC images by stitching specific rows/columns from a row of pinhole images. We mount a Cannon 60D SLR with 50mm F1.8 lens on translation track. Two planes are placed in front of the camera and the planes intersect with the camera path. We record a video while the camera is moving at a constant velocity. The resolution of the captured video is $1280 \times 720$. From each frame, we choose a specific column or row and stitch them together to form a new image. For pushbroom, we choose column 480 in all frames, as showed in Fig. 7 (b). We linearly increase the column index in terms of frame number and stitch these columns to form an XSlit image, as showed in Fig.7( (c). Finally, we linear increase the row index in terms of the frame number and stitch these rows to form a pencil camera, as showed in Fig. (7)d). The highlighted rectangles illustrate where CCPs occur in pushbroom, XSlit and pencil cameras, which is consistent with our theory.

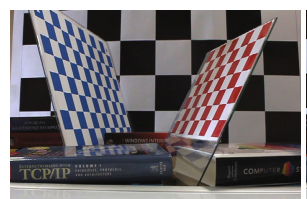

(a) Perspective

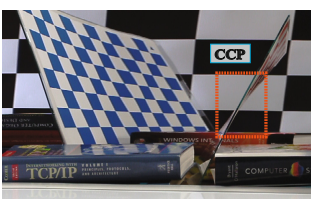

(b) Pushbroom

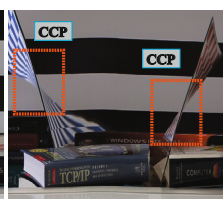

(c) XSlit

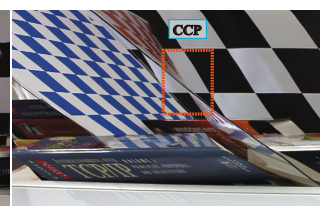

(d) Pencil

Fig. 7. Synthesized GLC images through stitching pinhole images. (a) The perspective view of the scene. (b) Pushbroom Image. (c) XSlit Image. (d) Pencil Image. Notice lines on a common 3D plane coverage t CCP in Pushbroom, XSlit and Pencil images.

Concentric Mosaics. To construct a concentric mosaic, we mount a Cannon 60D SLR with $50 \mathrm{~mm}$ F1.8 lens on a rotation table. We align the optical axis to pass through the rotation axis. Two planes are placed in front of the camera and the planes intersect the camera path. We record a video as the camera rotates. The system setup is showed in Fig. 8 (a). From the recorded view, we select specific columns from different frames and stitch them to form a concentric mosaic image. Fig. 8 b) shows the images formed by stitching column 560 from all frames. Fig. 8. (c) shows the result by stitching column 840. The highlighted regions show where the CCPs occur. 


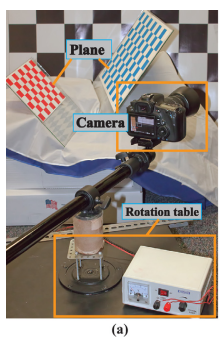

(a)

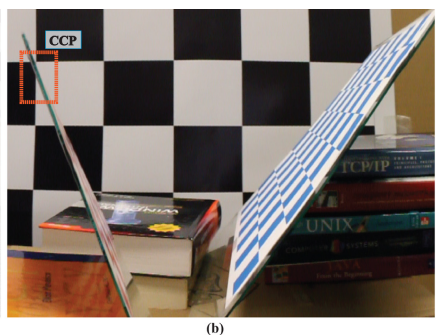

(b)

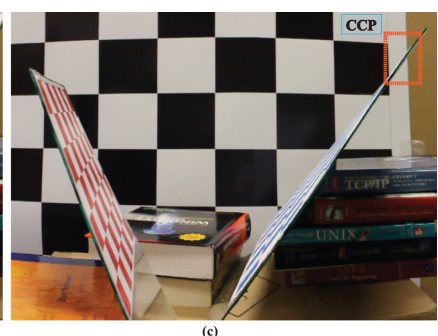

(c)

Fig. 8. Captured CCP in concentric mosaics

Cylindrical Mirror. We place a PointGrey FL2-08S2C camera in front of a cylindrical mirror and align the optical axis with the cylindrical axis, as showed in Fig. 9(a). The resolution of the captured images is $1024 \times 768$. We place a plane in front of the mirror and the catadioptric images of the plane is shown in Fig. 9(b) and (c). Notice that the plane exhibits the CCP. To understand why this is the case, recall that our analysis shows that as far as the plane has an intersection with the caustic circle, the plane generally has a CCP. In our case, we position the plane near the center of the mirror and tilt it so that it is guaranteed to intersect with the circle and hence has a CCP.

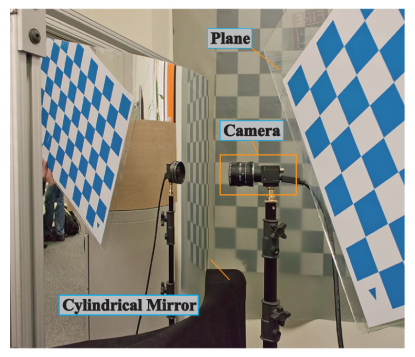

(a)

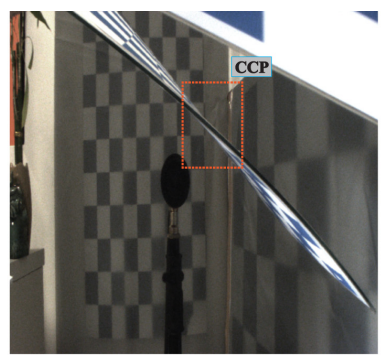

(b)

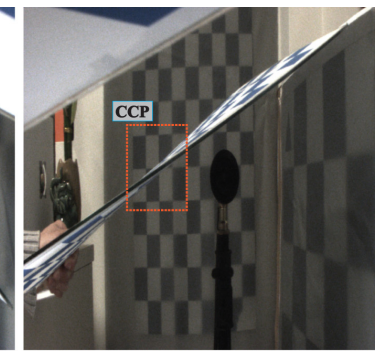

(c)

Fig. 9. Captured CCP in cylinder mirror

Spherical Mirror. Our analysis shows that catadioptric systems based on rotationally symmetric mirrors generally cannot capture the CCP of a plane. In fact, even the CCP does exist, it can lie outside the image plane. We have developed a curve-fitting algorithm to handle this case and details can be found in the supplementary materials. In our experiment, we first mount a spherical mirror on a vertical reference plane. The radius of the mirror is $51.64 \mathrm{~mm}$. We place the PointGrey FL2-08S2C camera with focal length $7.85 \mathrm{~mm}$ in front of the spherical mirror. The camera is pre-calibrated. Next, we align the optical axis to pass through the center of sphere using the reference plane, as showed in Fig. 10 (b). We set the center of the spherical mirror to be the origin of the coordinate system and connect it with the camera's $\mathrm{CoP}$ as the $z$ axis. As a result, the $\mathrm{CoP}$ is at $[0,0,182.37]$ in our coordinate system.

We attach three parallel white stripes on to a black plane and place it in front of the mirror. The captured image is showed in Fig. 10(a). We then apply our curve fitting algorithm of the white stripes and the fitted results are showed in Fig. 10 (c). Our results reveal that the images of these stripes (lines) intersect at two CCPs in 


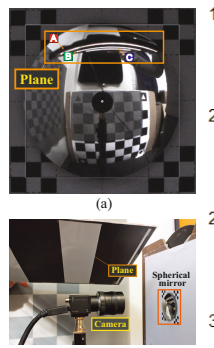

(b)

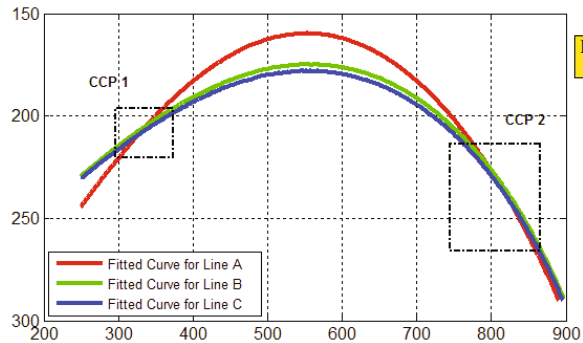

(c)

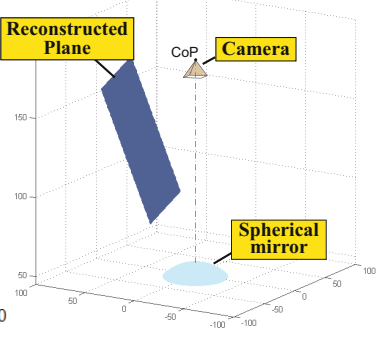

(d)

Fig. 10. Line projection in a symmetric catadioptric mirror. Left: We show the line image and the mirror profile; Middle: Located CCPs by curve fitting; Right: Reconstructed plane by using CCPs.

addition to the vanishing points. The 3 fitted curves, however do not exactly intersect at the same CCPs due to errors in curve fitting. We therefore average the estimation as the final detected CCPs. In this example, the two CCPs have pixel coordinate as $[332.5,207.1]$ and $[809.45,232.5]$. We map them back to their reflection points on the mirror at $z^{*}=33.675$ and finally locate the plane from the two CCPs and $z^{*}$. The plane reconstructed is $x+6.2806 y-3.8944 z+131.14=0$, showed in Fig. 10(d).

\section{Conclusions and Discussions}

We have explored a new type of image features called the coplanar common point or CCP in general non-centric cameras. A CCP corresponds to the intersection of the curved projections of all lines lying on a common 3D plane. We have shown that CCPs generally exist in a broad range of non-centric cameras such as the general linear camera, and the perspective camera is the single exception that do not have CCP. We have further derived the necessary and sufficient conditions for a plane to have CCP in an arbitrary non-centric camera such as non-centric catadioptric mirrors. Our experiments have validated our theories and the detected CCPs can facilitate 3D plane localization tasks, which is crucial to $3 \mathrm{D}$ scene reconstruction.

There are several future directions we plan to explore. First, the accuracy of CCP largely depends on curve fitting. Since the curves in catadioptric mirrors are of a highorder, our current solution is to search for the optimal solution from a set of basis functions using a look-up table. In particular, we do not consider the mirror geometry when fitting curves and as a result, it can be sensitive to discretization errors. In the future, we plan to develop tailored curve fitting algorithm by imposing mirror geometry as constraints. In addition, although our caustic-based analysis is applicable to arbitrary catadioptric mirrors, we have by far only studied in depth the cylindrical and rotationally symmetric mirrors. In the future, we will explore efficient testing schemes for general catadioptric mirrors. Finally, we intend to integrate the VP and CCP analysis under a unified geometric framework. Conceptually VPs present directions and CCPs represent positions. A unified representation under projective geometry [11] may sufficiently address both problems via a more elegant model. 
Acknowledgements. This project was partially supported by the National Science Foundation under grants IIS-CAREER-0845268 and IIS-1218156.

\section{References}

1. Baker, S., Nayar, S.K.: A theory of single-viewpoint catadioptric image formation. IJCV 35(2) (1999)

2. Caglioti, V., Gasparini, S.: "How many planar viewing surfaces are there in noncentral catadioptric cameras?" towards singe-image localization of space lines. In: 2006 IEEE Computer Society Conference on Computer Vision and Pattern Recognition, vol. 1, pp. 1266-1273 (June 2006)

3. Delage, E., Lee, H., Ng, A.Y.: Automatic single-image 3D reconstructions of indoor manhattan world scenes. In: ISRR (2005)

4. Gupta, R., Hartley, R.I.: Linear pushbroom cameras. IEEE TPAMI, 963-975 (1997)

5. Hoiem, D., Efros, A.A., Hebert, M.: Geometric context from a single image. In: ICCV (2005)

6. Hoiem, D., Efros, A.A., Hebert, M.: Automatic photo pop-up. In: ACM SIGGRAPH (2005)

7. Levoy, M., Hanrahan, P.: Light field rendering. In: ACM SIGGRAPH, pp. 31-42 (1996)

8. Meingast, M., Geyer, C., Sastry, S.: Geometric models of rolling-shutter cameras. CoRR (2005)

9. Pajdla, T.: Stereo with oblique cameras. In: IEEE Workshop on Stereo and Multi-Baseline Vision, pp. 85-91 (2001)

10. Pajdla, T.: Geometry of two-slit camera. Rapport Technique CTU-CMP-2002-02, Center for Machine Perception, Czech Technical University, Prague (2002)

11. Ponce, J.: What is a camera? In: The IEEE Conference on Computer Vision and Pattern Recognition (2009)

12. Shum, H.Y., He, L.W.: Rendering with concentric mosaics. In: Proceedings of the 26th Annual Conference on Computer Graphics and Interactive Techniques, pp. 299-306. ACM Press/Addison-Wesley Publishing Co. (1999)

13. Sturm, P., Ramalingam, S., Tardif, J.P., Gasparini, S., Barreto, J.: Camera models and fundamental concepts used in geometric computer vision. Foundations and Trends in Computer Graphics and Vision 6(1-2), 1-183 (2011)

14. Swaminathan, R., Grossberg, M., Nayar, S.: Caustics of Catadioptric Cameras. In: IEEE International Conference on Computer Vision (ICCV), vol. 2, pp. 2-9 (July 2001)

15. Swaminathan, R., Grossberg, M.D., Nayar, S.K.: Non-single viewpoint catadioptric cameras: Geometry and analysis. Int. J. Comput. Vision 66(3), 211-229 (2006)

16. Tom, H.B.: Rendering novel views from a set of omnidirectional mosaic images. In: Proceedings of Omnivis 2003: Workshop on Omnidirectional Vision and Camera Networks. IEEE Press (2003)

17. Ye, J., Ji, Y., Yu, J.: Manhattan scene understanding via xslit imaging. In: CVPR (June 2013)

18. Yu, J., McMillan, L.: Modelling reflections via multiperspective imaging. In: IEEE Computer Society Conference on Computer Vision and Pattern Recognition, CVPR 2005, vol. 1, pp. 117-124 (June 2005)

19. Yu, J., McMillan, L.: General linear cameras. In: Pajdla, T., Matas, J. (eds.) ECCV 2004. LNCS, vol. 3022, pp. 14-27. Springer, Heidelberg (2004)

20. Yu, J., McMillan, L., Sturm, P.: Multiperspective modeling, rendering, and imaging. In: ACM SIGGRAPH ASIA 2008 Courses, pp. 14:1-14:36 (2008)

21. Zomet, A., Feldman, D., Peleg, S., Weinshall, D.: Mosaicing new views: the crossed-slits projection. IEEE TPAMI 25(6), 741-754 (2003)

22. Zomet, A., Feldman, D., Peleg, S., Weinshall, D.: Non-perspective imaging and rendering with the crossed-slits projection. Tech. rep., Leibnitz Center, Hebrew University of Jerusalem (2002) 